\title{
Motion Triggers Indefinite Stoppage Against Binocular Rivalry
}

\author{
Ahmad Yousef ${ }^{1}$ \\ ${ }^{1}$ School of Computational Science and Engineering, McMaster University, Hamilton, Ontario, Canada \\ *Correspondence: mohamas2@mcmaster.ca
}

\begin{abstract}
This article provides evidence that high-speed high-information visual stimulus is able to indefinitely dominate the visual awareness; at speed of 21 degrees/second. Greater speeds emphasize greater dominance duration for the motion stimulus, however, at 21 degrees/second the moving stimulus dominates the visual awareness exclusively and indefinitely. Astonishingly, no room for mixed precepts had been reported by the human subjects at that speed, as if, it's the ultimate domination.

Introduction

Commonly agreed, visual motion stimuli had been always elongating the dominance duration in binocular rivalry settings, (Malek, 2012). More importantly, coherent motion shrinks human pupil size (Sahraie, et al. 1997). Additionally, in aging studies, it had been reported that pupil size becomes smaller with age (Sloane, etal. 1988); moreover, the dominance duration in binocular rivalry settings is getting greater with age, (Ukai, etal. 2003). Interestingly enough, constricted pupils assist in rapid shifts in the foveal image, these rapid foveal shifts emphasizing elongation of the dominance duration, (van Dam etal. 2006). Needless to mention, bottom-up stream of information may increase the dominance duration when presented either continuously (Blake etal., 2005) or abruptly (Blake etal., 2006; Reynolds etal., 2004). Based on all of the aforementioned facts, we hypothesize that deceeding a certain threshold of pupil constriction along with rapid presentation of massive stream of bottom-up information might elongate the dominance duration, as a possible result of the busy conscious brain. In another word, deceeding certain threshold of pupil constriction might significantly elongates the dominance duration, and indefinitely cease the rivalry. After cautiously taken the dynamics between the pupil and the lens into our consideration to ultimately comprehend the human visual awareness, see reference 8 , it looks like pupil constriction intensify the rapid changes of the foveal images of the moving stimuli, (van Dam etal. 2006). We think that; constricted pupil might be a signal from the eye to tell us that the neurological roads "the links that connect the photoreceptors with their corresponding terminal neural nodes 'the inhibitory neurons' which eventually might signal the consciousness" are currently busy or possibly partially idle, see reference 9 . Namely, the pupils might constrict as defensive mechanism against the external
\end{abstract}

visual information, to reduce the congestion in the 'busy' neurological roads. In another word, the congested neurological roads might be suffering to timely deliver the visual information to consciousness; and thus, the pupil might receive an indirect order to constrict for congestion management. To validate our hypothesis, we had created special stimuli that we believe it carries significant amount of visual information (see rainbow strip, reference 10). We also let that stimulus to run in various speeds, namely, zero, seven, and twenty-one degrees/seconds, see reference 10. At the highest speed, astoundingly, the binocular rivalry had been indefinitely stopped, and the moving stimulus exclusively dominates the visual awareness, with no room for the stationary stimulus nor mixed percepts. The possibly unstoppable pupil constriction might be an indicator of very congested 'neurological roads' in the brain. Since the moving stimulus will continue to dominate the consciousness, we might state that; this is ultimate visual awareness involuntary domination!

Materials \& Methods

Twelve human subjects, with average pupils, were recruited to participate in this study. Each stimulus subtends exactly a circle of 8.76 DVA, namely, 3.7 centimeters, on the screen, whereas the human subjects are 24 centimeters away from the screen, wearing 9.25 diopters base-out glasses, see reference 10. Each human subject has to undergo six trials, namely, two trials (original, and counterbalancing) for each of the following speeds, stationary, seven degrees/second, and 21 degrees/second; see reference 10. For quantitative analysis, human subjects had used a specialized timer for recording every switch, namely, key press 1 for right-sided stimulus, key press 2 for the left-sided stimulus, and key press 3 for mixed percepts. While collecting the behavioral data, since the fast-moving stimuli cause rapid pursuit eye movements; and to comply to Helsinki declaration, we set all of the trails to be for five minutes each. We believe that five minutes of unstoppable visual awareness domination for the fast-moving stimulus may be considered as indefinite stoppage against binocular rivalry.

Results and Discussion

Difference of dominance duration, as shown in the following figure is the dominance duration of motion 
stimulus minus the dominance duration stationary stimulus. In fact, we applied (inverse $\log _{10}$ ) to the aforementioned difference to be able to visualize the huge effect. The steps to calculate the aforementioned values are as follows, we first estimated the average dominance duration for each stimulus per each human subject. The twelves values out of the human subjects produces twelve different mean values for each trail, these values are used to estimate an overall mean value and the standard error of the mean value for the difference between motion stimulus awareness minus stationary stimulus awareness. Important to mentioned, each trial lasts five minutes. As shown in the infographic, high-speed high-information stimulus were able to stop the rivalry for its entire trail, namely, five minutes of stoppage. Important to mention, all of the human subjects had reported negligible mixed precepts for the low-speed stimuli, but none of them had reported mixed percept for either stationary or high-speed stimuli. It is an essential to reminder that $G A B A$ concentration has a positive correlation with the dominance duration of binocular rivalry stimuli (van Loon et al., 2013). Lorazepam, a stimulator of $G A B A_{A}$ receptors, had shown a similar effect (van Loon et al., 2013); as if there is a positive correlation between viewing high-speed, high-information stimuli and the activation of GABA machinery. Importantly, we have previously theorized that the inhibitory neurons had been sending information to the consciousness, and the consciousness has spectrum, starting from oblivion and ending with metacognition, see reference, 9. Based on van Loon et al., 2013 previous findings; indefinite stoppage against binocular rivalry might require indefinite 'local' activations of GABA machinery that exceed the human biological capabilities; and this might never happen. Our entanglement theory, thus, might have just obtained a further virtuous evidence. Namely, we might conclude that 'the high-speed, high information visual stimulation' stimulus influences the consciousness in a way that enables the awareness to continuously build entangled wave functions between the dominant stimulus and its corresponding photoreceptors, mainly because indefinite GABA machinery 'local' activation might be biologically impossible. Important to mention, the first trial to stop the rivalry indefinitely was done by Olivia Carter and her colleagues; which was accomplished by meditation of Tibetan Buddhist monks. In this letter, however, we had offered an involuntary procedure that emphasize indefinite stoppage against binocular rivalry. The aforementioned facts might conditionally invalidate (van Loon et al., 2013)'s positive correlation between $G A B A$ concentration and dominance duration; and thus, might provide rigorous credibility to our entanglement theory; however, no confirmation can be authorized without retinal studies. Essential Notification

Stimuli and the BR setup can be

found in reference 10. Readers who don't have binocular rivalry setup are welcome to visit our lab. Due to the stimuli complexity, BR setup is a must to properly experience the effect, otherwise, interference problems may occur. Scholars with brain imaging and retinal imaging facilities are welcome to cooperate. Enquiries should be sent to the author. Transactional References

[1] Malek, etal. (2012). Binocular rivalry of spiral and linear moving random dot patterns in human observers.

[2] Sahraie, and Barbur (1997) "Pupil response triggered by the onset of coherent motion." Graefes Arch Clin Exp Ophthalmol. [3] M, Sloane, C. Owsley, and S. Alvarez (1988). "Aging, senile miosis and spatial contrast sensitivity at low luminance". Vision Research.

[4] Ukai, etal. (2003). Binocular rivalry alternation rate declines with age. Perceptual and Motor Skills.

[8] van Dam LC, van Ee R (2006a) Retinal image shifts, but not eye movements per se, cause alternations in awareness during binocular rivalry. Journal of vision.

[5] Chong, Tadin, \& Blake (2005). Endogenous attention prolongs dominance durations in binocular rivalry. Journal of Vision.

[6] Chong, \& Blake (2006). Exogenous attention and endogenous attention influence initial dominance in binocular rivalry. Vision Research.

[7] Mitchell, Stoner, \& Reynolds (2004). Object-based attention determines dominance in binocular rivalry. Nature.

[8] Yousef, A. 2019. "Linking Dynamics Between Pupil and Lens." engrXiv. doi:10.31224/osf.io/9c3vk.

[9] Yousef, Ahmad. 2019. "Consciousness Might Be Localized in Extra Physical Dimensions." PsyArXiv. doi:10.31234/osf.io/angc8.

[10] Stimuli and the BR system may be found here: https://drive.google.com/drive/folders/1ETD3_-H6p_tmaDqmvBHXHExsi_7KrqQ? usp= sharing

[11] van Loon et al., (2013). GABA shapes the dynamics of bistable perception. Current Biology.

[12] O. Carter, etal. (2005). Meditation alters perceptual rivalry in Tibetan Buddhist monks. Current Biology. 